\title{
3 Research Square

\section{A nomogram to predict stricture after endoscopic submucosal dissection for early esophageal cancer}

\section{Xue Li}

Beijing Friendship Hospital

Jie Xing

Beijing Friendship Hospital

Peng Li

Beijing Friendship Hospital

Shengtao Zhu

Beijing Friendship Hospital

Qian Zhang

Beijing Friendship Hospital

Shutian Zhang ( $\nabla$ zhangshutian@ccmu.edu.cn )

Beijing Friendship Hospital

\section{Research Article}

Keywords: Esophageal stricture, Endoscopic submucosal dissection, Risk factor, Nomogram

Posted Date: August 10th, 2021

DOI: https://doi.org/10.21203/rs.3.rs-733195/v1

License: (c) (i) This work is licensed under a Creative Commons Attribution 4.0 International License.

Read Full License 


\section{Abstract}

Background: Risk factors of esophageal stricture after ESD are inconsistent and incomprehensive, the present study aimed to identify a robust model to predict postoperative stricture.

Methods: This retrospective study included 273 individuals underwent ESD for early esophageal cancer from January 1, 2014 to October 30, 2020 in Beijing Friendship Hospital. Univariate Cox proportional hazard regression was used to estimate potential factors of stricture after ESD, followed by multivariate Cox proportional hazard regression to identify significant independent predictors after adjusting for covariates. Nomogram building based on multivariate Cox regression model was used to evaluate accuracy and discrimination by ROC curves and calibration curves respectively.

Results: Stricture was identified in 28 patients (10\%). The other patients were enrolled in non-stricture group $(n=243)$. Multivariate Cox proportional hazard model demonstrated that operation time (HR 1.01, 95\%Cl: 1.00-1.01, $P<0.01$ ), upper segment of esophagus (HR 6.48, 95\%Cl: 2.07-20.33, $P<0.01$ ), middle segment of esophagus (HR 3.42, 95\%Cl: 1.36-8.64, $P<0.01$ ), $>3 / 4$ circumferential lumen of esophagus (HR 6.32, 95\% Cl: 2.20-18.13, $P<0.01$ ), postoperative fever (HR 6.17, 95\% Cl: 1.75-21.77, $P<0.01$ ), were independent predictive factors for postoperative stricture. The predictive accuracy of the nomogram was estimated by the AUC of ROC curves was $0.93,0.87,0.87$ at 1 year, 2 years, and 3 years respectively.

Conclusion: Longer operation time, $>3 / 4$ of the circumferential lumen of esophagus, located at upper and middle esophageal segment, and postoperative fever were independent risk factors of esophageal stricture after ESD.

\section{Background}

Early esophageal cancer is expected to undergo en bloc resection using endoscopic submucosal dissection (ESD), making the 5-year survival rate high as $92.0 \%(95 \% \mathrm{Cl}, 83.1-95.9)[1,2]$. Stricture formation is one of the major adverse effects associated with ESD, causing serious dietary deficiencies that reduce the quality of life and necessitate multiple endoscopic balloon dilatation (EBD) sessions that incur high medical costs[3]. Multivariate logistic analysis revealed that more than $3 / 4$ of the circumferential extension is a reliable risk factor[4]. But little consensus has been achieved in longitudinal resection length $>50 \mathrm{~mm}$, the depth of infiltration more than lamina propria (m2), muscular injury and clip number and so on[5-7]. Postoperative symptoms and laboratory tests were seldom incorporated into models. The aim of this study is to more comprehensively analyze the independent risk factors associated with postoperative stricture and provide a robustly ready-to-use clinical prediction model to select high-risk people.

\section{Materials And Methods}

\section{Patients}


This was a retrospective cohort study consecutively collected 361 cases completed ESD for early esophageal cancer from 1st January 2014 to 30th October 2020 at Beijing Friendship Hospital, Capital Medical University in China. Exclusion criteria included: (1) not high-grade intraepithelial neoplasia (HGIN)/squamous cell carcinoma (SCC) confirmed by histopathology; (2) additional treatment due to positively cutting edge of resected specimen, such as surgery treatment, radiotherapy and chemotherapy; (3) missing the gastroscopy review or other essential information.

\section{Information and materials}

Information of patient characteristics (age, sex, drinking history, smoking history, body mass index (BMI), family history), lesion characteristics (infiltration depth) and treatment process (operation time, hospital stay, glucocorticoid prevention, postoperative fever, blood test) was collected from medical record. Endoscopic images of lesions (lesion location, longitudinal lesion length, proportion of esophageal circumference, number of clips, postoperative stricture) was collected from picture archiving and communication system (Qingdao Medicon Digital Engineering Co., Ltd., Medicare I-EndoCenter, Qingdao, China).

Esophageal stricture was defined as an esophageal diameter $<11 \mathrm{~mm}$, rather than the inability to pass the gastroscope (which had a diameter of 9.8-11.0 mm)[8]. Classify dysphagia severity into the following four grades: score $0=$ able to eat a conventional diet; score 1 (mild)=able to eat some solid meals; score 2 ( moderate)=able to eat semisolid but not solid foods; score 3 (severe) =able to consume liquids only; score $4=a p h a g i a[9]$. Score 2 and above was defined as both dysphagia and esophageal stricture.

The upper segment of esophagus was defined as the cervical and upper thoracic segments, $15-25 \mathrm{~cm}$ away from the incisors; the middle segment of the esophagus was defined as mid-thoracic esophagus, $25-30 \mathrm{~cm}$ away from the incisors; the lower segment of esophagus was defined as the lower thoracic esophagus and the abdomen, below $30 \mathrm{~cm}$ from the incisors[10]. Operation time was defined as duration of anesthesia. Postoperative fever was defined as axillary body temperature exceeding $37.3^{\circ} \mathrm{C}$ within 48 hours after ESD. White blood cell (WBC) was tested on the second day after ESD. Deep muscle layer injury, subcutaneous emphysema, mediastinal emphysema, and esophageal fistula were defined as perforation. Some patients with large resected area were prescribed to oral glucocorticoid (Tianjin Lisheng Pharmaceutical Co., Ltd., Prednisone acetate, Tianjin, China) to prevent stricture, with a dose of $30 \mathrm{mg} /$ day on the third day after ESD, tapered gradually $(30,30,25,25,20,15,10$, and $5 \mathrm{mg}$ for 7 days each) and then discontinued 8 weeks later[11].

\section{Esophageal ESD}

A complete description of the ESD (Olympus, Co., Ltd., GIF-Q260J/GIF-Q180, Tokyo, Japan) procedure has been reported[12]. Mark margin of the lesion with dual knife, inject Meilan hyaluronate sodium saline into submucosa, cut the periphery, gradually peel off the lesion along the submucosa, and intermittently give hemostatic forceps to stop bleeding. Careful observation of no active bleeding on the wound, indwelling gastric tube after operation. 


\section{Study endpoints}

Endoscopic review was performed 3th month and 9th month after ESD and once a year thereafter or when a patient complained of dysphagia associated with semisolid foods. The primary endpoint was esophageal diameter $<11 \mathrm{~mm}$ or dysphagia confirmed. The second endpoint was scarring confirmed or the last gastroscopy review.

\section{Statistical analysis}

The student $t$ test was used to compare the differences between continuous variables with normal or approximate normal distribution, which were presented as the mean and standard deviations (SD). Other continuous variables were presented as the median and quartile, and the categorical variables were presented as counts and percentages. Chi-square (c2) test was for unordered categorical variables. The Mann-Whitney test were used to compare ordinal categorical variables or continuous variables that not meet Student's test. Univariate Cox regression analysis was used to evaluate the effects of clinically significant variables. Statistically significant factors obtained from the univariate Cox regression analysis were then entered into a forward LR multivariate Cox proportional hazard regression to identify independent risk factors after adjusting for other covariates. The risk factors for stricture formation were estimated by calculating the hazard ratios (HR) and the $95 \%$ confidence intervals $(95 \% \mathrm{Cl})$. The statistical tests above were evaluated by SPSS software version 22.0.

Kaplan-Meier cumulative incidence plots were created by Graphpad Prism (v.9.1.1.225). Package "rms" "survivalROC" in R Software 4.0.5 (www.r-project.org) was used for nomogram building based on multivariate Cox proportional hazard regression model. Receiver operating characteristic curves (ROC) were used to present the predictive accuracy of nomograms graphically. We performed calibration for established nomograms and applied 1,000 repetitions by 4 groups $(n=55)$ of bootstrap sample corrections to internally validate the nomograms. The area under the curve (AUC) for validation was applied to evaluate the accuracy of the nomogram. Two-sided and a value of $P<0.05$ was considered statistically significant all above.

\section{Results}

\section{Clinical Characteristics}

Retrospectively collect 361 patients performed ESD for early esophageal carcinoma in Friendship hospital endoscopy center from January 1st, 2014 to October 30th, 2020. A total of 90 individuals were excluded, 271 patients were eligible into the study, stricture was identified in 28 patients (10\%) and the rest in non-stricture group (243, 90\%) (Figure 1). The median follow-up time was 116 (96-191) days.

The baseline characteristics (age, sex, drinking and smoking history, BMI, family history) had no statistical difference between two groups ( $P>0.05)$. Infiltration depth of lesion and perforation were not significantly different $(P>0.05)$. Whereas lesion location, involved esophageal circumference, lesion 
length, operation time, number of clips, hospitalization stay, glucocorticoid prevention, postoperative fever and WBC count had significantly statistic difference between the two groups $(P<0.05)($ Table 1$)$.

\section{Predictive factors for postoperative stricture}

Univariate Cox proportional hazard regression showed that operation time, longitudinal lesion length, lesion location, proportion of esophageal circumference, perforation, postoperative fever, glucocorticoid prevention and WBC count may be clinically risk factors of postoperative stricture (Table 2). Forward LR multivariate Cox proportional hazard regression analysis on the basis of the former demonstrated that operation time (for 1-minute increase) (HR 1.01, 95\% Cl: 1.00-1.01, $P<0.01$ ), upper segment of esophagus (HR 6.48, 95\%Cl: 2.07-20.33, $P<0.01$ ), middle segment of esophagus (HR 3.42, 95\% Cl: 1.36-8.64, $P<0.01$ ), $>3 / 4$ circumference involved (HR 6.32, 95\%Cl: 2.20-18.13, $P<0.01)$, postoperative fever (HR 6.17, 95\%Cl: 1.75-21.77, $P<0.01$ ), could be independent predictive factors for postoperative stricture (Table 2). The Kaplan-Meier curves showed that the stricture cumulative incidence was higher among more than $3 / 4$ circumference of esophagus group (c2=49.14, $P<0.01$; Figure $2 \mathrm{~A}$ ), located upper and middle segment of esophagus group ( $22=5.03, P=0.03$; Figure $2 \mathrm{~B}$ ) and postoperative fever group (c2=20.36, $P<0.01$; Figure 2C).

\section{Nomogram for the prediction of esophageal postoperative stricture}

We developed a predictive nomogram from the multivariate Cox proportional hazard regression model (Fig 3A). Calibration curves generated by 1,000 repetitions of bootstrap sample corrections (4 groups, $\mathrm{n}=55$ ) were illustrated good for 1 year, 2 years and 3 years respectively (Fig 3B-D). ROC curves were drawn to estimate the predictive accuracy of the nomogram, and the AUC was $0.93,0.87,0.87$ at 1 year, 2 years, and 3 years respectively (Fig 3E-F).

\section{Discussion}

Evidence of this study showed that more than 3/4 circumferential esophageal lumen, lesions near upper esophagus, postoperative fever and operation time were the independent risk factors of esophageal stricture after ESD. >3/4 luminal circumference was consistent with the vast majority of previous studies, while the conclusion of other three risk factor varied from literature to literature[7,13].

Nomograms as estimators have shown promising potential in clinical trial design and interpretation and have been widely adopted in prognostic models[14-16]. To our knowledge, this is the first study providing a nomogram to predict postoperative stricture risk. ROC curves and calibration curves suggested that the nomogram is a powerful tool in individual risk assessment. Refractory stricture is mostly formed within 1 year after ESD, so that there was little difference between the risk predictions for 2 years and 3 years in the nomogram chart and calibration curves. We also took glucocorticoid prevention effect to stricture into consideration. The incidence $(38 \%, 15 / 39)$ of esophageal stricture in patients with resection lesion $>3 / 4$ circumference was obviously lower than the $88-100 \%$ incidence of stricture in other studies[13,17]. So we 
speculated that oral small glucocorticoid had not a statistic effect on the prevention due to not enough sample size in our study[11,18,19].

Lesions in the upper and middle segment of esophagus $(P<0.01)$ were independent risk factors for postoperative stricture. This might be related to esophagus anatomy that the diameter of upper and middle segment smaller than lower segments besides narrow sites[20]. In addition, the symptoms of dysphagia caused by the narrowing of stricture in the upper segment appeared earlier and more obviously. Similar report suggested that lesion in the cervical segment is an independent risk factor for postoperative stricture[21]. But in our study only one lesion located in the cervical esophagus, so we classified it as the upper segment of esophagus.

Influential factors of longer operation time for ESD included location, gross type, tumor depth, ulcerative findings, lesion size, endoscopist experience and so on[22]. The more difficult it was to remove the lesion, the longer the operation time needed. Multivariate regression analysis was used to eliminate the mixed interference of factors such as large circumferential ratio, location, clip number and perforation. And all the endoscopists performing ESD in our center are experienced chief professor. The longer time surgery took, the more frequent intraoperative hemorrhage and endoscopic electrocoagulation, leading to extensive fibrosis and scar formation related to postoperative stricture.

It was demonstrated that postoperative fever was an independent factor for esophageal stricture in our study, which was never analyzed in previous study. Some literature illustrated fever is a common phenomenon during the first 48 hours after surgery, which is mostly non-infectious[23,24]. This is regulated by pyrogenic cytokines such as serum interleukin-l, interleukin-6, tumor necrosis factor, and interferon-gamma. The more tissue damage, the greater the cytokine release[24]. We speculated that patients after esophageal ESD suffered fever maybe for major invasive procedures so that they were more likely to develop postoperative stricture.

This study has a number of strengths. Complete collection of detailed clinical data and outcomes, and use of multivariable, time-varying analyses to quantify independent risk factors for esophageal stricture with a relatively large sample size so far. A nomogram tool was established to select the population for early prevention based on Cox regression model. By the way, fever within 48 hours after ESD could be a simple and effective predictor in addition to the circumference ratio, which was not found in previous studies.

This study has several limitations. First, it took place in single hospital in Beijing, China, potentially limiting generalizability to other places and we lack enough data to do external validation of the nomogram model so far. Second, our study was a retrospective cohort study, there were inevitably problems of recall bias and a small part of missing data such as tumor infiltration depth which was been proved to be a risk factor in previous studies[5,21]. Therefore, a prospective controlled study with a higher level of evidence and external validation of the nomogram model would be needed to verify our conclusions. 


\section{Conclusion}

Our study proved longer operation time, $>3 / 4$ circumference of the esophageal lumen, lesion location, and postoperative fever were significantly associated with stricture after ESD. Nomogram, a ready-to-use predictive tool, was provided with the potential to select high-risk people with postoperative stricture. But this is a single-center retrospective study, further studies are necessary to verify whether our findings generalize to other patients.

\section{Abbreviations}

ESD: endoscopic submucosal dissection; EBD: endoscopic balloon dilatation; m2: lamina propria; HGIN: high-grade intraepithelial neoplasia; SCC: squamous cell carcinoma; WBC: White blood cell; SD: standard deviations; c2: Chi-square; HR: hazard ratios; $95 \% \mathrm{Cl}$ : 95\% confidence intervals; ROC: receiver operating characteristic curves; AUC: area under the curve; TP: true positive rate; FP: false positive rate

\section{Declarations}

\section{Ethics approval and consent to participate}

This study was approved by the Institutional Ethics Committee of Beijing Friendship University, Capital Medical University $\mathbb{}$ Beijing $\triangle$ China [2020-P2-290-01]. All procedures performed in this study using human data were in accordance with the 1964 Helsinki declaration and its later amendments or comparable ethical standards. Informed consent was waived by the Institutional Ethics Committee of Beijing Friendship University, Capital Medical University because of the retrospective nature of this study and all of the data from medical record.

\section{Consent for publication}

Not applicable.

\section{Availability of data and material}

The datasets used and analyzed during the current study available from the corresponding author on reasonable request.

\section{Competing interest}

The authors declare that they have no competing interests.

\section{Funding}

This work was fully supported by the Digestive Medical Coordinated Development Center of Beijing Hospitals Authority (grant no. XXZ01 and XXZ02). The funders had no role in study design, data collection and analysis, decision to publish, or preparation of the manuscript. 


\section{Authors' contributions}

$\mathrm{XL}, \mathrm{JX}, \mathrm{PL}$ and SZha conceived and designed the study. Data collection and analysis were performed by $\mathrm{XL}, \mathrm{JX}, \mathrm{QZ}$ and SZhu. The first draft of the manuscript was written by XL. And SZha proofread and revise the manuscript. All authors commented on the draft of the manuscript. All authors read and approved the final manuscript.

\section{Acknowledgements}

Not applicable.

\section{References}

1. Takahashi $\mathrm{H}$, Arimura $\mathrm{Y}, \mathrm{Masao} \mathrm{H}$, et al. Endoscopic submucosal dissection is superior to conventional endoscopic resection as a curative treatment for early squamous cell carcinoma of the esophagus (with video). Gastrointest Endosc. 2010;72(2):255-64, 264.e1-2.

2. Nagami $Y$, Ominami $M$, Shiba $M$, et al. The five-year survival rate after endoscopic submucosal dissection for superficial esophageal squamous cell neoplasia. Dig Liver Dis. 2017;49(4):427-433.

3. Ono H, Yao K, Fujishiro M, et al. Guidelines for endoscopic submucosal dissection and endoscopic mucosal resection for early gastric cancer (second edition). Dig Endosc. 2021;33(1):4-20.

4. Amamoto Y, Kikuchi D, Nagami Y, et al. Management of adverse events related to endoscopic resection of upper gastrointestinal neoplasms: Review of the literature and recommendations from experts. Dig Endosc. 2019;31(Supp I):4-20.

5. Shi Q, Ju H, Yao LQ, et al. Risk factors for postoperative stricture after endoscopic submucosal dissection for superficial esophageal carcinoma. Endoscopy. 2014;46(8):640-4.

6. Tang J, Kong F, Li J, et al. Independent risk factors for esophageal refractory stricture after extensive endoscopic submucosal dissection. Surg Endosc. 2020; doi: 10.1007/s00464-020-07840-w.

7. Chen $M$, Dang $Y$, Ding $C$, et al. Lesion size and circumferential range identified as independent risk factors for esophageal stricture after endoscopic submucosal dissection. Surg Endosc. 2020;34(9):4065-71.

8. Takahashi H, Arimura Y, Okahara S,et al. A randomized controlled trial of endoscopic steroid injection for prophylaxis of esophageal stenoses after extensive endoscopic submucosal dissection. BMC Gastroenterol. 2015; 22(15):1.

9. Knyrim K, Wagner HJ, Bethge $\mathrm{N}$, et al. A controlled trial of expansile metal stent for palliation of esophageal obstruction due to inoperable cancer. N Engl J Med. 1993;329(18):1302-7.

10. Sami SS, Haboubi HN, Ang Y, et al. UK guidelines on oesophageal dilatation in clinical practice. Gut. 2018;67(6):1000-23.

11. Yamaguchi $\mathrm{N}$, Isomoto $\mathrm{H}$, Nakayama $\mathrm{T}$, et al. Usefulness of oral prednisolone in the treatment of esophageal stricture after endoscopic submucosal dissection for superficial esophageal squamous 
cell carcinoma. Gastrointest Endosc. 2011;73(6):1115-21.

12. Bahin FF, Jayanna M, Hourigan LF, et al. Long-term outcomes of a primary complete endoscopic resection strategy for short-segment Barrett's esophagus with high-grade dysplasia and/or early esophageal adenocarcinoma. Gastrointest Endosc. 2016;83(1):68-77.

13. Ono S, Fujishiro M, Niimi K, et al. Predictors of postoperative stricture after esophageal endoscopic submucosal dissection for superficial squamous cell neoplasms. Endoscopy. 2009;41(8):661-5.

14. Kim SK, Chai YJ, Park I, et al. Nomogram for predicting central node metastasis in papillary thyroid carcinoma. J Surg Oncol. 2017;115(3):266-72.

15. Nie Z, Zhao P, Shang Y, et al. Nomograms to predict the prognosis in locally advanced oral squamous cell carcinoma after curative resection. BMC Cancer. 2021;21(1):372.

16. Zhao F, Lu RX, Liu JY, et al. Development and validation of nomograms to intraoperatively predict metastatic patterns in regional lymph nodes in patients diagnosed with esophageal cancer. BMC Cancer. 2021;21(1):22.

17. Funakawa K, Uto H, Sasaki F, et al. Effect of endoscopic submucosal dissection for superficial esophageal neoplasms and risk factors for postoperative stricture. Medicine (Baltimore). 2015;94(1):e373.

18. Oliveira JF, Moura EG, Bernardo WM, et al. Prevention of esophageal stricture after endoscopic submucosal dissection: a systematic review and meta-analysis. Surg Endosc. 2016;30(7):2779-91.

19. Kataoka M, Anzai S, Shirasaki T, et al. Efficacy of short period, low dose oral prednisolone for the prevention of stricture after circumferential endoscopic submucosal dissection (ESD) for esophageal cancer. Endosc Int Open. 2015;3(2):E113-7.

20. Oezcelik A, DeMeester SR. General anatomy of the esophagus. Thorac Surg Clin. 2011;21(2):289-97.

21. Yu X, Liu Y, Xue L, et al. Risk factors for complications after endoscopic treatment in Chinese patients with early esophageal cancer and precancerous lesions. Surg Endosc. 2021;35(5):2144-53.

22. Goto O, Fujishiro M, Kodashima S, et al. Is it possible to predict the procedural time of endoscopic submucosal dissection for early gastric cancer? J Gastroenterol Hepatol. 2009;24(3):379-83.

23. O'Grady NP, Barie PS, Bartlett JG, et al. Guidelines for evaluation of new fever in critically ill adult patients: 2008 update from the American College of Critical Care Medicine and the Infectious Diseases Society of America. Crit Care Med. 2008;36(4):1330-49.

24. Maday KR, Hurt JB, Harrelson P, et al. Evaluating postoperative fever. JAAPA. 2016;29(10):23-8.

\section{Tables}

Due to technical limitations, Table 1 and 2 are only available as a download in the Supplemental Files section.

\section{Figures}




\section{ESD for Early Esophageal Cancer $(n=361)$}

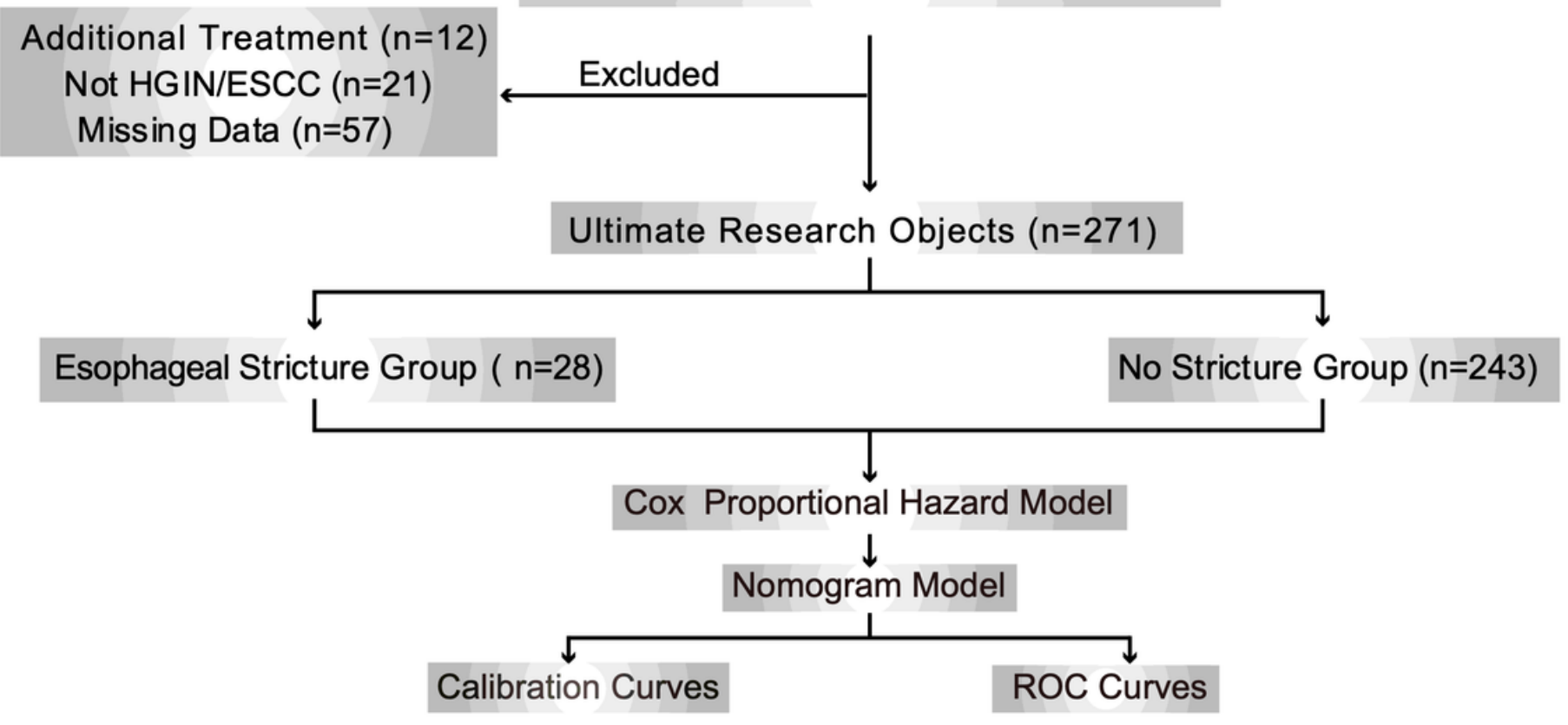

Figure 1

Flow chart of the study. 361 patients underwent esophageal ESD, 90 patients were excluded for additionally therapy $(n=12)$, and not HGIN/SCC histopathology $(n=21)$, and incomplete information $(n=57) .271$ patients were included in the Cox regression model, visualized through nomogram, and evaluated for accuracy (ROC curves) and discrimination (Calibration curves).
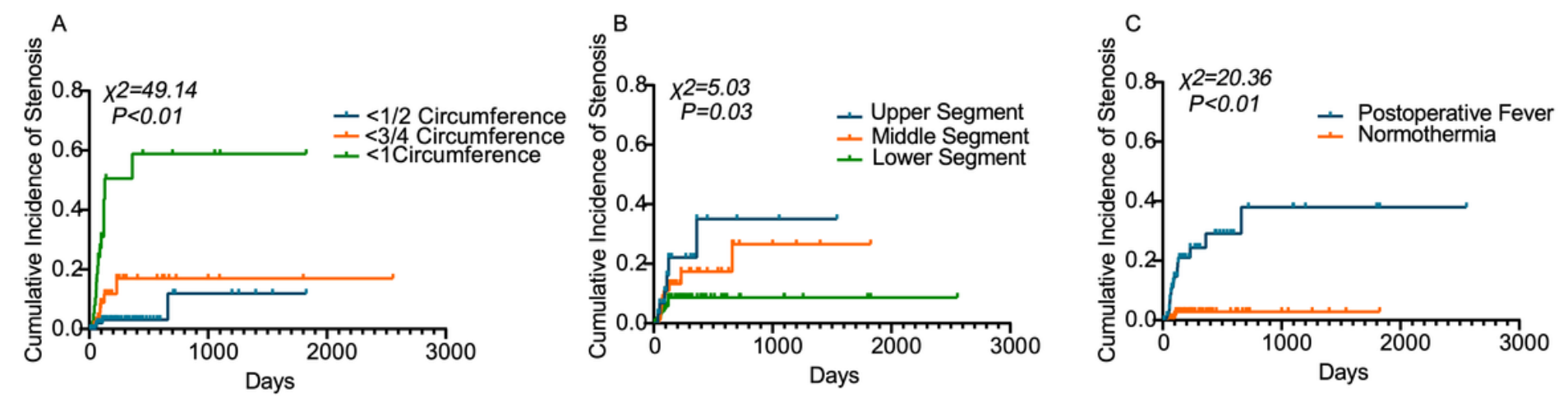

\section{Figure 2}

Kaplan-Meier cumulative incidence curves. (A) Different proportional esophageal circumference developed postoperative stricture. (B) Lesion location developed postoperative stricture. (C) Postoperative fever or not developed postoperative stricture. 
A

Points

$\begin{array}{lllllllllll}0 & 10 & 20 & 30 & 40 & 50 & 60 & 70 & 80 & 90 & 100\end{array}$

Circumference

Location

Operation Time

Postoperative Fever

Total Points

$<1 / 2 \quad$ Middle
Lower

1-year Predictive Risk

2-year Predictive Risk

3-year Predictive Risk

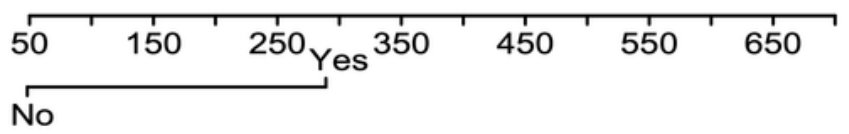

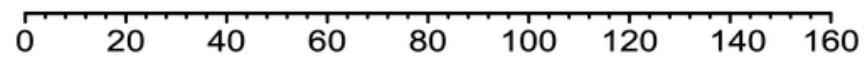

$$
\begin{array}{llllllll}
0.1 & 0.3 & 0.5 & 0.7 & 0.9
\end{array}
$$$$
\begin{array}{llllllll}
0.1 & 0.3 & 0.5 & 0.7 & 0.9
\end{array}
$$
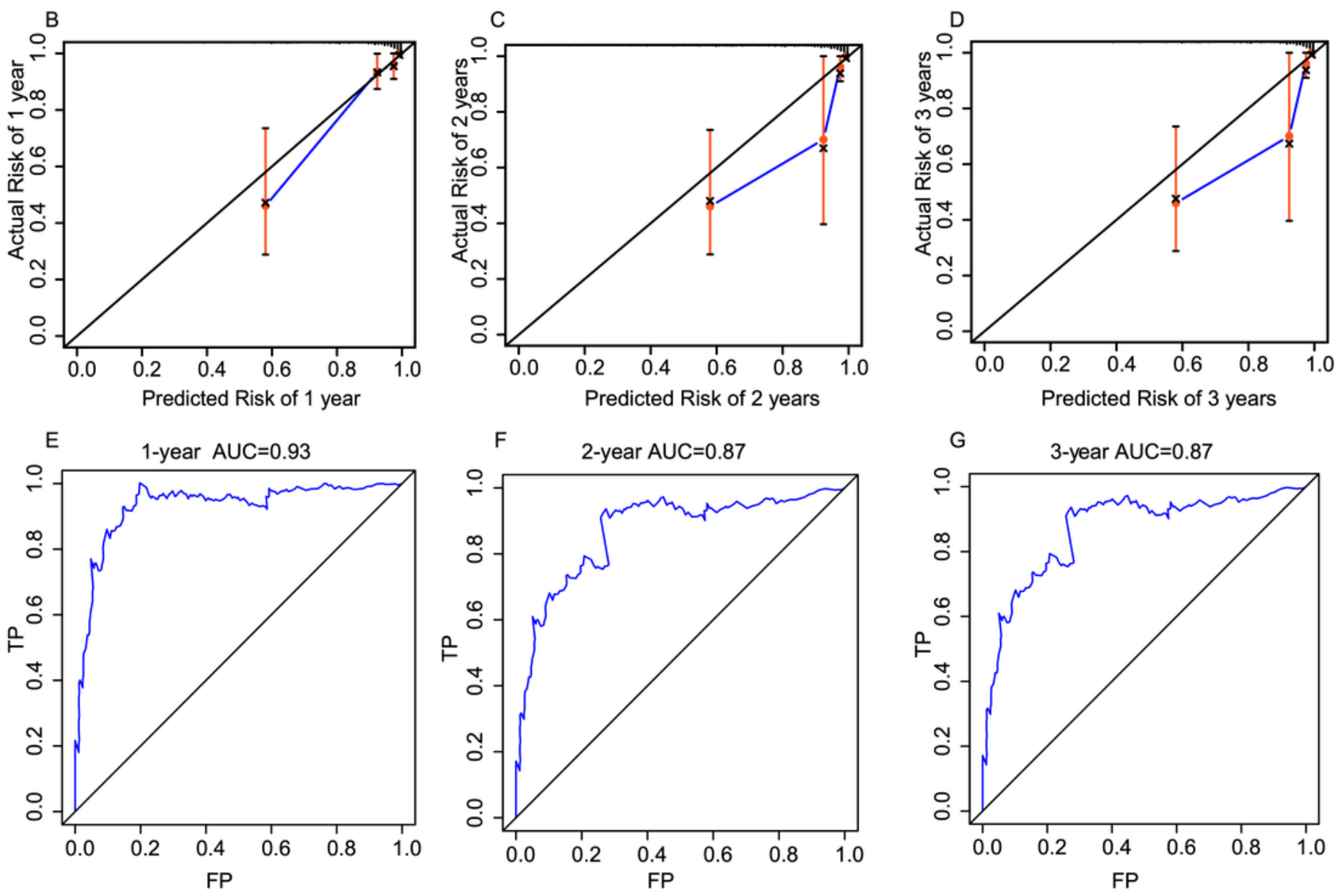

\section{Figure 3}

(A) A nomogram prediction model of stricture after esophageal ESD. (B-D) Calibration curves of this nomogram model for 1 year, 2 years and 3 years respectively. (E-G) ROC curves to predict overall risk incidence at 1 year, 2 years and 3 years respectively using this nomogram model. 
This is a list of supplementary files associated with this preprint. Click to download.

- Table1.docx

- Table2.docx 